Théologiques

Théologiques

\title{
Le rien et le féminisme
}

À propos de la gratuité

\section{Denise Couture}

Volume 4, numéro 2, octobre 1996

L'épreuve du rien

URI : https://id.erudit.org/iderudit/602442ar

DOI : https://doi.org/10.7202/602442ar

Aller au sommaire du numéro

\section{Éditeur(s)}

Faculté de théologie de l'Université de Montréal

\section{ISSN}

1188-7109 (imprimé)

1492-1413 (numérique)

Découvrir la revue

\section{Citer cet article}

Couture, D. (1996). Le rien et le féminisme : à propos de la gratuité.

Théologiques, 4(2), 99-115. https://doi.org/10.7202/602442ar
Résumé de l'article

Le rien apparaît d'emblée comme un de ces concepts étrangers, même rébarbatifs, aux théories féministes. Luce Irigaray l'a abordé dans son essai sur Martin Heidegger. Elle établit un lien entre la manière dont il construit le rien et la néantisation du 'féminin' dans le discours. En son projet discursif, il logerait 'elle' au lieu de la gratuité même, du don qui se donne sans limite de ressource et sans retour. Mais 'elle' est aussi ailleurs, celle qui parle et qui invite à un échange. Le chemin de l'homme vers le rien et la gratuité pourrait ne plus habiter le langage de l'indifférence sexuelle. d'utilisation que vous pouvez consulter en ligne.

https://apropos.erudit.org/fr/usagers/politique-dutilisation/ 
Théologiques 4/2 (1996) 99-115.

\section{Le rien et le féminisme}

\section{À propos de la gratuité}

Denise COUTURE

Faculté de théologie Université de Montréal

\section{RÉSUMÉ}

Le rien apparaît d'emblée comme un de ces concepts étrangers, même rébarbatifs, aux théories féministes. Luce Irigaray l'a abordé dans son essai sur Martin Heidegger. Elle établit un lien entre la manière dont il construit le rien et la néantisation du 'féminin' dans le discours. En son projet discursif, il logerait 'elle' au lieu de la gratuité même, du don qui se donne sans limite de ressource et sans retour. Mais 'elle' est aussi ailleurs, celle qui parle et qui invite à un échange. Le chemin de l'homme vers le rien et la gratuité pourrait ne plus habiter le langage de l'indifférence sexuelle.

Nothingness has appeared at once to feminist theories as an unfamiliar and daunting concept. Luce Irigaray has tackled this theme in her essay on Martin Heidegger. She establishes a link between the manner in which he constructs nothing and negation of the "feminine * in discourse. In his discursive project he would locate "she " in the realm of " gratuitousness* itself, of a gift that gives itself without restraint of ressources and without return. But " she * is also somewhere else, she who speaks and who invites exchange. The route of man towards nothingness and gratitude can no longer inhabit the language of sexual indifference.

Le projet de tenir discours à partir d'une position d'énonciation féministe m'a appris que le sexe n'est pas indifférent. Et qui plus est à propos de la gratuité. Femmes et hommes n'éprouveraient pas la gratuité de la même manière. En première approximation, le masculin semble avoir besoin de se former à plus de gratuité; de cesser de tout calculer pour son propre intérêt et de pouvoir fournir partout des raisons; d'apprendre le don de soi, le service, l'amour. Le féminin, au contraire, exprimerait déjà un trop-plein de gratuité; aurait besoin de mettre en question la gratuité 
naturelle qu'on lui attribue; de lutter pour une rémunération équitable, pour une reconnaissance du travail d'éducation des enfants et du soin des autres; de désapprendre le service tel que si bien appris, en excès. De là, l'hypothèse qu'elle et lui auraient des défis différents (inverses?) à relever sur les chemins des constructions culturelles de la gratuité; d'où l'hypothèse, également, qu'il ne pourrait me suffire, qu'il ne pourrait suffire à une parole féminine et féministe, de s'insérer dans les discours masculins, sinon au péril de représenter leur problème spécifique et d'occulter la différence sexuelle.

Le mot gratuité signifie trois choses : - qui ne coûte rien (sens économique), - action désintéressée (sens moral) et - qui est sans raison, sans fondement (sens philosophique). Chacune des trois définitions comporte des privatifs marquant la négation : les adverbes ne... rien, le préfixe dés, la préposition sans. La gratuité ne semble se donner à concevoir que par les abords de sa périphérie; que par une description des pourtours de son domaine, au nombre de trois : l'échange donnant-donnant, la quête d'intérêt, le fait de fournir des raisons. Les définitions négatives de la gratuité indiquent qu'elle $n$ 'est pas soumise à la logique des causes et des effets du temps linéaire de l'horloge. Ce n'est pas agir 'en conséquence de', 'parce que' ou 'en vue de'. Elle est le sans prix, le sans intérêt, le sans raison.

Comment cela arrive-t-il? Comment dire la positivité de la gratuité? Le « sans », la négation, la néantisation adviennent-ils différemment pour le 'féminin' et pour le 'masculin'? Comment la perspective importe-t-elle?

Ou encore : peut-on penser la gratuité sous le mode même du gratuit? Faut-il inévitablement poser la question de sa valeur, montrer son intérêt, expliciter la raison du "sans raison "? Quelles voies d'accès tracer pour la penser? Deux voies?

Gratuité : ce qui est sans raison, sans fondement.

Je partirai, dans ce qui suit, de cette troisième définition, métaphysique, des dictionnaires. Car elle inclut les deux autres. Le "sans raison" implique déjà qu'il n'y a ni raison d'échange, ni raison d'intérêt.

On se tournera d'abord volontiers vers le penseur du sans-fond, Martin Heidegger, pour méditer une sentence telle 'Ce qui est sans raison, sans fondement'. Mais les femmes font-elles une expérience du sansfond différente de celle des hommes (Ab-grund)? Appartiennent-elles, appartiennent-ils différemment à la gratuité? Le penseur jugerait-il ces questions inappropriées, du moins, précipitées, en regard de la tâche de penser l'être en tant qu'être? Ces questions me conduisent, dans cet article, à présenter deux parcours de la gratuité, en vue d'un dialogue, 
celui de Martin Heidegger et celui de la théoricienne féministe Luce Irigaray.

\section{Un fil conducteur : « La rose est sans pourquoi »}

Dans sa lettre d'invitation à contribuer à ce cahier de Théologiques, Jean-Claude Petit m'écrivait :

Le langage se souvient d'une autre dimension du rien, qui le fait elle aussi échapper à nos prises mais en ouvrant devant, cette fois, une contrée lumineuse bien que tout aussi mystérieuse et inquiétante.

Il nommait cette contrée celle du "don pour rien ", de la gratuité, et rappelait une parole d'Angelus Silesius, suggérant une piste, par la voie poétique : "La rose est sans pourquoi. Elle fleurit parce qu'elle fleurit. Elle ne fait pas attention à elle-même, ne demande pas si on la regarde. "

Ces vers sont connus par Heidegger qui les commente dans son livre Le principe de raison. Elle est tirée d'un recueil de poésies spirituelles, publié pour la première fois en 1657, et intitulé Le Pelerin chérubinique. Description sensible des quatre choses dernières. Johann Scheffler (1624-1677) était docteur en philosophie et en médecine; ses textes de poésie, sous la plume d'Angelus Silesius, étaient cités par Leibniz et Hegel qui leur reconnaissaient un dire mystique ${ }^{1}$.

La rose est sans pourquoi, fleurit parce qu'elle fleurit.

N'a souci d'elle-même, ne désire être vue.

La sentence donne à penser, en effer, en direction du "don pour rien ", de la gratuité. Mais, comme le souligne Heidegger, une rose n'est pas un étant à la mesure du Dasein. Contrairement à la rose, celui-ci a à penser, chaque fois, ce qu'il a à être; il a souci de lui-même. Le penseur cherche tout de même à entendre les résonances de la sentence en tant qu'elle ne vise pas à "faire ressortir la différence de l'homme et de la rose", tout en maintenant une différence essentielle entre la rose et l'être-la $a^{2}$. Comment le Dasein est-il quand il est sans pourquoi? Le commentaire, par Heidegger, de la sentence d'Angelus Silesius nous introduit, en effet, directement à une méditation de 'ce qui est sans raison'.

1 Voir Martin HEIDEGGER, Le principe de raison. Paris, Gallimard, 1957, p. 103 104. Je cite la traduction de la sentence dans cette édition.

$2 \quad$ lbid, p. 115. « Bien courte à vrai dire serait notre pensée, si nous admettions que la sentence d'Angelus Silesius n'a d'autre sens que d'indiquer la différence des manières dont la rose, dont l'homme, sont ce qu'ils sont ", p. 108. 
Avant de lire ce commentaire, il convient cependant encore de préparer une voie d'accès au questionnement; de ne pas laisser de côté, déjà, la préoccupation de la différence sexuelle, précédemment énoncée. Le premier vers commenté par le philosophe, 'La rose est sans pourquoi, fleurit parce qu'elle fleurit', est placé, par Luce Irigaray, en exergue de son livre sur Martin Heidegger, L'oubli de l'air $(1983)^{3}$, et le projet de la théoricienne féministe vise à penser un occulté, partout et toujours, dans la formation philosophique: la différence sexuelle. Dans son essai sur Heidegger, elle pense, à partir de là, "la rose sans pourquoi " et montre comment s'organise la néantisation d'elle chez le penseur. Luce Irigaray cherche, elle aussi, une autre pensée, mais qui serait portée par l'oubli de la différence sexuelle. Pour le dire avec Rosi Braidotti, le projet consiste à tenir que « juste comme la mort, la différence sexuelle est toujours déjà là, qu'on le reconnaisse ou non 4 .

\section{Heidegger et la différence sexuelle}

Silence de Heidegger sur la différence sexuelle? Jacques Derrida a noté que

du sexe, oui, on le remarque facilement, Heidegger parle aussi peu que possible et peut-être ne l'a-t-il jamais fait. Peut-être n'a-t-il jamais rien dit, sous ce nom, sous les noms que nous leur connaissons, de 'rapport-sexuel', de la 'différence-sexuelle', voire de 'l'homme-et-lafemme's.

Silence qui en dit beaucoup, selon Luce Irigaray; silence apparent, selon Jacques Derrida. Comme le souligne celui-ci, à lire Heidegger, tout se passe comme si

une différence sexuelle n'était pas à hauteur de différence ontologique : aussi négligeable en somme, au regard de la question du sens de l'être, qu'une différence quelconque, une distinction déterminée, un prédicat ontique. Négligeable s'entend pour la pensée, même si elle ne l'est en rien pour la science ou pour la philosophie. Mais en tant qu'il s'ouvre à la question de l'être, en tant qu'à l'être il a

3 Luce Irigaray, L'oubli de l'air chez Martin Heidegger. Paris, Éd. de Minuit, 1983, $157 \mathrm{p}$.

4 Rosi BraidotTI, Nomadic subjects. Embodiment and sexual difference in contemporary feminist theory. New York, Columbia University Press, 1994, p. 131.

5 Jacques DERRIDA, « Différence sexuelle, différence ontologique (Geschlecht I) ", dans: Heidegger et la question. Paris, Flammarion, 1990 (1987), p. 147. Dans les prochains paragraphes, je renvoie à cet ouvrage. 
cette référence même le Dasein ne serait pas sexifère. Le discours sur la sexualité serait ainsi abandonné aux sciences ou aux philosophies de la vie, à l'anthropologie, à la sociologie, à la biologie, peut-être même à la religion ou à la morale. (pp. 147-148, souligné dans le texte).

Les questions entourant la différence sexuelle distrairaient de la question directrice, celle de l'être. Au plus, apparaîtraient-elles comme des sous-questions thématiques à l'intérieur du projet de l'ontologie existentiale du Dasein. Elles n'auraient pas la force d'affecter le penser de l'essentiel. L'énoncé, par Heidegger, de la neutralité du Dasein viserait à réduire, à soustraire " toute prédétermination anthropologique, éthique ou métaphysique pour ne garder qu'une sorte de rapport à soi, de rapport dépouillé à l'être de son étant " (p. 152). Si le Dasein n'est pas l'humain (Mensch), à plus forte raison n'est-il ni la femme ni l'homme, d'où sa neutralité sexuelle.

Apparemment? Une analyse d'un texte moins cité de Heidegger 6 conduit Derrida à voir et à entendre dans cette négativité anthropologique une positivité originaire que Heidegger lui-même n'a pas vu et n'a pas entendu. L'analyse dévoile un dire sur le sexe qui se camoufle en non-dits. Elle démonte les mécanismes, voire le besoin, de ce camouflage. L'hypothèse du lecteur français : la différence sexuelle appartiendrait à la structure ontologique du Dasein ("Serait-ce là une interprétation trop violente? ", écrit-il). Est-ce à dire qu'il y aurait deux "là "? Deux rapports à l'être, au rien et au sans raison?

Ce type d'analyse pourrait être poursuivie, le là-féminin pensé, son appartenance à la gratuité, au sans-fond, méditée dans sa différence. Luce Irigaray ajoute à cette stratégie une autre voie. Parce que, pour elle, la racine de l'effacement d'elle est plus profonde. Il ne s'agit plus, comme chez le collègue Jacques Derrida, de rendre le penseur sauf, de montrer qu'il va plus loin qu'il ne le pense lui-même, que la différence sexuelle appartient à la structure ontologique du là, qu'il ne reste qu'à prolonger le chemin déjà tracé et à penser le là-féminin. Il s'agit plutôt de faire 'apparaître' la néantisation du la-elle comme condition de possibilité du projet de penser l'être-là et l'être en tant qu'être. Luce Irigaray montre comment l'occultation de la différence sexuelle permet la pensée même de Heidegger

6 Où il explicite le projet de Sein und Zeit et y précise la fonction qu'y occupe le Dasein. Cours d'été, 1928. "Metaphysische Anfangsgründe der Logik im Ausgang von Leibniz ", Gesamtausgabe, t. 26. 
et comment on pourrait penser autrement ${ }^{7}$. Elle aborde cette pensée comme un mode de vie, comme une manière de respirer, construite par 'lui'. La marque féministe de son approche est de poser que le discours neutre a toujours déjà approprié le 'féminin'.

\section{Lire Heidegger à partir d'une énonciation féministe}

Les théories féministes mettent à l'épreuve plus d'une stratégie constructive d'une position d'énonciation. Elles prennent appui, il le faut, sur différents épistèmes qui nient 'elle'. Malgré leur différence épistémique, cependant, elles ne jouent pas les unes contre les autres ainsi que les théories auxquelles elles réfèrent ${ }^{8}$. Dans ce qui suit, je me laisserai guider par Luce Irigaray, pour penser la gratuité et, également, pour éprouver le mode d'analyse féministe qu'elle met en œuvre.

Elle ne procède ni par fuite, à côté ou en dehors, du discours masculin, ni par contra-diction.

Il n'est, dans un premier temps, peut-être qu'un seul 'chemin', celui qui est historiquement assigné au féminin : le mimétisme. Il s'agit d'assumer, délibérément, ce rôle. Ce qui est déjà retourner en affirmation une subordination, et, de ce fait, commencer à la déjouer. Alors que récuser cette condition revient, pour le féminin, à revendiquer de parler en 'sujet' (masculin), soit à postuler un rapport à l'intelligible qui maintient l'indifférence sexuelle ${ }^{9}$.

La question est de savoir comment penser la différence sexuelle alors que le 'féminin' demeure profondément enfoui dans la nuit de la neutralité du discours. Par la voie de ce même discours? Oui, répond Luce Irigaray, car il n'y a d'autre voie pour commencer à parler. Elle situe l'enjeu de l'indifférence sexuelle au niveau de discours dont 'elle' ne s'est jamais évadée mais en lequel 'elle' peut s'insérer stratégiquement. La voie proposée, le mimétisme délibéré, implique trois éléments.

7

Rosi Braidotti a raison : La recherche d'un penser autrement " a figuré à l'ordre du jour du projet philosophique depuis Heidegger; et il me semble que le féminisme en tant que mouvement de pensée soit pris dans cette problématique et qu'il ait un rôle majeur à y jouer ", Nomadic Subjects, p. 134.

$8 \AA \grave{A}$ propos, par exemple, du rapport 'non contradictoire' entre essentialisme et constructivisme en théorie féministe, voir Naomi SCHOR, "Introduction ", dans : Naomi SCHOR et Élizabeth WEED (dir.), The essential difference. Indianapolis, Indiana University Press, 1994, pp. vii-xix; pour une analyse plus large des relations entre les théories, spécifiques aux féminismes, voir Rosi BRAIDOTTI, “Women's studies and the politics of difference ", dans : Nomadic Subjects, pp. 205-212.

9 Luce IrIGARAY, Ce sexe qui n'en est pas un. Paris, Éd. de Minuit, 1977, pp. 73-74. 
D'abord, 'elle' n'a pas de lieu d'où parler. Le sol, donné par lui, à elle, à partir d'où penser la différence sexuelle serait-il, dès l'origine, projeté hors champ du penser? Alors d'où parler? Elle n'a pas de lieu où habiter avec lui, le langage construit par le penseur; pas de lieu d'où le contredire, hormis son archi-langage qui l'a déjà appropriée. " Je n'ai pas encore trouvé le lieu d'où je pourrais commencer à dire quoi que ce soit. Ici maintenant ", écrit Luce Irigaray dans son commentaire de la pensée de Heidegger ${ }^{10}$. La tâche de l'énonciation féministe est, chaque fois, la construction d'une position d'énonciation.

Ensuite, 'elle' s'insère dans le discours à partir de la place à elle assignée, de la position qu'elle occupe déjà, donnée par lui. Afin de la démonter. Le mimétisme délibéré tire sa force de ce que les femmes habitent aussi ailleurs. Elles peuvent ainsi ne pas simplement reproduire le même du discours masculin. "Jouer de la mimésis", écrit Luce Irigaray, c'est "pour une femme, tenter de retrouver le lieu de son exploitation par le discours, sans s'y laisser simplement réduire ${ }^{11}$ \%. Le mimétisme opère comme une insertion dans le discours qui vise la construction d'une position-elle d'énonciation, mais par l'occupation de la position (subordonnée) qu'assigne le texte au féminin, pour la détourner.

Enfin, 'elle' se conjugue au conditionnel. 'Elle' serait, aurait, pourrait, éprouverait... des possibilités de changement d'air(s). "Que, là où elle demeure déjà, le paraître et son dire ne soient pas encore, ne signifie pas qu'elle demeure dans la nuit ${ }^{12}$ ». 'Elle' peut parler au mode conditionnel; préparer, chaque fois, une respiration d'air, sachant que la préparation est déjà un souffle. Ce qui prévient de toute échéance sur la représentation. Il ne s'agit pas « d'élaborer une nouvelle théorie dont la femme serait le sujet ou l'objet, mais d'enrayer la machinerie théorique elle-même, de suspendre sa prétention à la production d'une vérité et un sens par trop univoques ${ }^{13}$ ". En un moment de pause, dans son texte sur Heidegger, Luce Irigaray fait retour sur ce qu'elle vient d'énoncer. "Est-ce vrai? »,

10

L'oubli de l'air, pp. 31-32. L'énoncé est tiré d'un paragraphe placé entre parenthèses.

11 Luce IrIGaray, Ce sexe qui n'en est pas un, p. 74. Et encore : c'est viser à « faire 'apparaître', par un effet de répétition ludique, ce qui devait rester occulté : le recouvrement d'une possible opération du féminin dans le langage " (p. 74).

12 Luce IrIGaray, L'oubli de l'air, p. 99. Le féminin recherché * est encore une page blanche, ce n'est pas encore là ", d'où, seulement, toujours à nouveau, une préparation à émerger au langage de la différence sexuelle (Rosi BRAIDOTTI, Nomadic subjects, p. 131, à propos de la méthode de Luce lrigaray).

13 Luce IRIGARAY, Ce sexe qui n'en est pas un, p. 75. Souligné dans le texte. 
demande-t-elle? "C'est difficile à dire. Quand je l'aurai ainsi nommée, cette opération sera déjà d'un autre ordre ${ }^{14}$ \%. La construction d'un lieu d'où parler est acte de construction qui pousse d'un conditionnel à un autre.

Penser la différence sexuelle vise à dé-neutraliser le neutre. Il s'agit de penser 'elle' dans une langue qui lui est étrangère; de penser "deux ": elle et lui, elles et eux, l'entre elles et l'entre eux. Luce Irigaray procède par l'insertion d'un je-elle, hypothétique, dans le discours, à partir du lieu où il l'a déjà appropriée. Où loge-t-elle la gratuité du 'féminin' dans l'archi-langage heideggérien? Comment Martin Heidegger aborde-t-il la gratuité, qui, de la perspective de la théoricienne, apparaîtra comme une gratuité du 'masculin'? De là, quelles pistes s'ouvrent pour repenser gratuité(s)? Je poserai ces trois questions, successivement, dans ce qui suit.

\section{4. «Producteur de gratuité entre ciel et terre ${ }^{15}$ »}

Le texte de Luce Irigaray n'est ni linéaire, ni argumentatif sous le mode d'un chemin tracé avec départ et arrivée. Il procède par traversées des lieux d'exil et d'enfermement où le penseur logerait 'elle'. Ainsi, en son rapport au texte du penseur, procède-t-elle par mimétisme délibéré.

J'essaie (...) de retraverser tous les lieux où j'ai été exilée-enfermée pour qu'il constitue son là. De lire son texte, pour tenter d'y reprendre ce qu'il m'a pris sans retour. De rouvrir tout ce qu'il a construit en me prenant dedans, en me mettant dehors, disant oui et non, ni oui ni non, me laissant dans un suspens d'attente et d'oubli où je ne puis vivre, bouger, respirer (p. 32).

Qui est 'elle' dans le texte du penseur? Où est-elle? Il ne le dit pas.

Qu'il la désigne ou non, l'appelle-rappelle ou non, la nomme ou non par exemple phusis, mais cela pourrait être autrement - n'y change rien. Peut-être même la fait-il ainsi entrer plus encore dans l'absence, et l'oubli (p. 45).

'Elle' serait 'nature', entre autres. 'Elle' serait : première matière, corps nourricier, don qui se donne sans retour et sans fin. Avec Luce Irigaray, femme, dans le discours, est gratuité même. Telle serait l'un de ses exils, de ses enfermements. Elle est le don sans limite de ressource et sans retour, une 'caractéristique' d'elle à comprendre d'abord comme une

14 Luce IrIGARAY, L'oubli de l'air, p. 76.

15 L'expression est de Luce IRIGARAY, dans L'oubli de l'air. Dans les paragraphes qui suivent, je renvoie à ce livre. 
opération qui nourrit le discours de l'indifférence sexuelle et le projet discursif construit par et pour l'homme. Un obstacle à la compréhension du texte de Luce Irigaray est de perdre de vue la méthode inhabituelle qu'elle met en jeu et qui consiste à faire émerger à la parole la vivante qui n'a pas de lieu dans le discours. La traversée du 'féminin' comme don premier de la vie vise à défaire l'évidence de la neutralité discursive.

$\grave{A}$ elle, donc, serait assignée la position du don sans limite et sans retour. "Elle donne d'abord ». Femme donne. "Elle donne - d'abord l'air, et sans retour, sinon que se déploie, à partir d'elle et en elle, qui lui prend de l'air » (p. 31). Ce qu'elle donne en premier, c'est :

la possibilité du commencement à partir duquel le tout de l'homme va se constituer. Ce don se reçoit sans retour. Il ne peut lui rendre la pareille. Les multiples et divers et incessants renvois qu'il y fait ou qu'il y fera n'auront jamais lieu au lieu du premier don. Une distance restera - infranchissable - entre ce là dont il provient et ses appels, rappels, renvois à... Le don - premier - reste sans 'réponse'. Ces deux-là ne communiquent que dans un sens: elle donne, il prend. Il n'y a pas, au commencement, de va-et-vient, d'aller-retour, du don (p. 31).

Blessure, pour lui, d'un impayable. Que fait-il? Il l'oublie. Il oublie sa provenance. Il construit l'être de l'être comme dernier commencement, comme sans-fond, de tout advenir et de toute existence. Ça se donne, ditil. Il construit le neutre. Il se donne. Qui? L'être. En excès. L'être voile le premier don; il exprime l'option d'un deuil de l'être-au-dehors d'elle.

Heidegger n'est pas seul en cette opération de neutralisation. Il dit lever le voile sur le commencement de la métaphysique, sur le destin de l'être. Avec Parménide. Il dit le commencement, oublié. Mais, à l'aurore, n'était-il pas déjà trop tard? L'être n'avait-il pas déjà recouvert l'oubli d"elle'? L'éveil du penseur à l'oubli de l'être ne la laisse-t-elle pas à sa nuit? Ne l'y enfonce-t-elle pas, une deuxième fois? Question trop lourde? Le projet de penser la différence sexuelle n'exigerait pas moins que celui de penser l'oubli de l'être, de repenser le tout du rapport au langage et au discours vers une autre pensée.

Le projet discursif de l'homme aurait déjà toujours approprié le 'féminin' et impliquerait une néantisation d'elle'. Il néantit celle qui pourrait commencer à parler et ainsi lui dérober le sans-fond de l'être; il se donne "un fondement à partir de la néantisation de ce dont il provient " (p. 93). L'être en tant qu'être serait le dernier commencement, la dernière provenance et le dernier énoncé, sans par-delà pensable. Mais, en cette opération, elle, qui donne d'abord, se trouve appropriée comme 'ce qui se donne'. Un double oubli : 
de celle qui lui a toujours déjà donné la vie et qui est devenue son corps vivant, de celle qui la lui redonne en l'assistant dans le destin de son être (p. 85).

Comment le penseur pourrait-il l'entendre? Est-ce seulement possible? Il s'est installé dans la sérénité de l'attention à l'être. L'homme construirait pour lui-même un abri, le sans-fond; et un style, la sérénité. Mais sa sérénité suppose "que rien ne reste: dehors"(p. 28). Il attend que le don se donne, il répond à la résonance de l'être, il laisse-être (Gelassenheit). Par une opération langagière, il se l'est assimilée. Le passage de l'un vers l'autre (l'une) n'est plus possible.

L'autre n'est plus que l'assimilation projetée dans le 'libre' du deuil de l'autre. Laisser être revient ainsi à abandonner à l'autre la garde de la sérénité devant l'absence du rapport entre l'un et l'autre - l'un et l'une (p. 27).

Son la, à elle, serait le "à partir d'où il y a du don ". Son là : un paslà-avec; mais ce qui s'efface en rendant possible tout advenir. 'Elle' est chaque fois là, mais jamais là parce que devenue sans-fond du projet de l'homme ${ }^{16}$.

"Producteur de gratuité ", écrit-elle : producteur d"elle' comme don dont il reçoit son projet. "Entre ciel et terre ", écrit-elle encore. Car de quoi est fait l'être de celui qui se dit le terrien? D'air ${ }^{17}$. Il a quitté le premier réceptacle pour naître à l'air. Il l'oublie. Il oublie l'air par une appropriation de ce dont il provient; y demeure en la logeant dedans-dehors. Ainsi, construit-il le rien comme provenance dernière : être de l'être.

En place de ce qui l'aurait abandonné, et vis-à-vis duquel il réitère le geste d'abandon, matrice de tout acte, l'homme se donne le néant. Le lien qui le reliait, comme engendré, à c'elle (sic) maternelle, se rompt. L'être peut exister comme un, se boucler en cercle (p. 92).

Le rien, comme provenance dernière du projet de l'homme, l'installe dans un retour incessant à son propre projet, à son même. Sans elle. En quoi ce rien? En air. Il se tiendrait " dans l'air libre " qu'il oublie.

Le fondement que l'homme se donne implique le voilement du néant sur lequel il repose. De la réduction à rien de ce dont, matière-chair, il provient. De la constitution en sans-fond de ce d'où il tire naissance (p. 146).

16 «Le don se donne sans entamer la réserve si celle qui ne reviendra plus est devenue, au présent, un transcendantal sensible toujours déjà et jamais plus là " (p. 87).

17 D'où le titre de l'ouvrage : L'oubli de l'air. 
C'est de là, de cette hypothèse de la néantisation d'elle' comme condition de la pensée de l'oubli de l'être, que Luce Irigaray entend la sentence de l'Ange de Silésie :

La rose est sans pourquoi, fleurit parce qu'elle fleurit.

N'a souci d'elle-même, ne désire être vue.

Qui est la rose? La position donnée, par lui, à elle? N'aurait souci d'elle-même? Appropriation de ce souci par lui? Elle ne serait pas en quête de raison. «Elle, n'en n'aurait pas besoin. Sa nécessité étant de fleurir » (p. 129). Elle serait hors champ de l'être-avec; 'première' matière et ressource où puiserait le projet discursif de l'homme. 'Elle' serait gratuité même, mais, aussi, ailleurs, la vivante.

Il a choisi de faire le deuil d'une séparation par la néantisation de l'autre, mais " elle peut encore lui révéler que sa vérité est la non-vérité de l'autre: son maintien dans l'absence d'un effroyable oubli" (p. 85). Qu'arrive-t-il si le penser se déplace? Penser l'être ou penser la différence sexuelle?

Si ce qui entre en elle ressort altéré d'avoir eu lieu en elle? (...) Alors, que devient l'être? (...) Si elle changeait d'air(s)? L'oubli s'oublierait comme tel? Donc, plus d'être? (p. 116).

La gratuité de celle qui parle pourrait ne plus se déployer dans la reproduction du là, à elle assignée, don sans limite de ressource, mais plutôt dans l'appel d'un échange entre elles et entre elle et lui, elles et eux. Quelles sont les conditions d'un tel échange?

\section{Heidegger et la rose}

La méditation, par Martin Heidegger, de la sentence d'Angelus Silesius s'offre en un tout autre style. Elle convie à un chemin de pensée vers le plus essentiel et je l'entendrai, avec Luce Irigaray, comme projet discursif de l'homme. Elle se trouve dans le livre Le principe de raison qui comporte treize leçons organisées en une architecture équilibrée ${ }^{18}$. Les six premières montrent les obstacles à une écoute authentique du principe de raison ${ }^{19}$, la septième résume le chemin parcouru et propose une reformulation de la question directrice, les six dernières s'engagent sur le

18 Cours donné à l'Université de Fribourg-en-Brisgau au semestre d'hiver 1955. 1956.

19 "Le fil conducteur suivi tout d'abord, écrit Heidegger, nous fait pour ainsi dire longer le principe du dehors ", Le principe de raison, p. 57. Dans les paragraphes qui suivent, je renvoie à ce livre. 
chemin de la nouvelle question qui, seule, permet le saut du penser. La méditation de la rose occupe une position charnière sur ce chemin. Elle se situe à la cinquième leçon. Heidegger y fait entendre deux résonances de la sentence d'Angelus Silesius. La première répond au parcours de la première partie du livre, la seconde introduit à l'autre rapport à la raison. Au dernier paragraphe du chapitre sur la rose, trouve-t-on, pour la première fois énoncée, la formule authentique du principe de raison: non plus "Rien n'est sans raison " (Tout a une raison), mais «Rien n'est sans raison 20 "(Être et raison : le même).

Une première écoute de la sentence, pour suivre le chemin de Heidegger, fait entendre que, contrairement à l'humain (Mensch ${ }^{21}$ ), la rose ne pose pas la question du "pourquoi ". Elle ne voit pas se glisser d'attention aux raisons, "en tant que raisons", entre sa floraison et les causes de sa floraison. L'humain, lui, peut découvrir et énoncer ces raisons. Le principe de raison, rien n'est sans raison, demeure ainsi valable, même pour la rose, en tant qu'elle est objet de la représentation humaine. La première résonance de la sentence confirmerait la forme rigoureuse du principe de raison tel que formulé par Leibniz: "Rien n'est sans une raison suffisante qui doit être fournie ". Le principe ne signifie pas que tout étant rende raison, mais que l'humain peut, partout et toujours, fournir raison en tant qu'il se représente un monde 22 .

Une deuxième écoute de la sentence provoque le revirement, le saut dans l'être en tant qu'être. Il semble, à première vue, que le "sans pourquoi $*$ de la rose nie la raison (en tant que la rose ne fournit pas de raisons) alors que le "parce que " l'affirme (en tant que l'humain peut donner les raisons de la floraison) 23 . Mais, «si nous y regardons mieux, écrit Heidegger, c'est l'inverse qui apparaît " (p. 113). Le "sans pourquoi » ne nie pas le rapport à la raison, mais pointe en direction d'un autre rapport, encore à méditer. Il montre seulement que «c'est de façons multiples que la raison peut être en rapport avec nous en tant que nous

"Raison ", ici, la traduction littérale de " ratio "; " Grund », en allemand, dont le premier sens est sol, fond et, de là, raison, fondement, motif, cause (voir p. 34, note de l'éditeur, et p. 65).

21 Il faut le noter, "Mensch", ici, est précisément marque de l'indifférence sexuelle, selon Luce Irigaray, de l'appropriation d"elle'.

22 Les six premières leçons du livre montrent comment la représentation et l'oubli de l'être reconduisent, par différents chemins, à cette interprétation.

23 Martin HEIDEGGER, Le principe de raison, p. 113. La rose ne demande pas les raisons; l'humain les indique. "'Pourquoi' sert à demander la raison. 'Parce que' répond et indique la raison ", (p. 105). 
sommes les êtres qui se représentent" (p. 114). Et le "parce que » n'affirme pas un 'rendre raison de'. Si nous écoutons bien, il ne dit rien, et, pour cela, il dit tout : « tout ce qui peut être dit de la raison et du pourquoi " (p. 115). Le "parce que " ne renvoie pas à quelque chose d'autre « qui ne serait pas un fleurir et qui devrait fonder la floraison à partir d'une altérité » (p. 141). Il renvoie à la floraison qui a son fondement, sa raison, en elle-même. La floraison est

pure éclosion hors de soi, pur éclat de ce qui brille. (...) La beauté est un mode suprême de l'être, c'est-à-dire ici : pure éclosion hors de soi, pur paraître et briller! (pp. 141-142).

La raison devient ici perceptible comme être et l'être comme raison $^{24}$.

Le revirement : le principe de raison ne dit pas que l'humain doive, chaque fois, courir après des raisons suffisantes; mais qu'être et raison sont une même chose ${ }^{25}$. D'où une nouvelle formulation du principe de raison qui guidera les six dernières leçons du penseur :

Sein und Grund: Dasselbe. Sein : Ab-grund

Être et raison : le même. Être : l'abîme

En sa nouvelle formulation, il faut le noter, le principe de raison inclut la définition de la gratuité. Avec Heidegger, penser l'un ou l'autre, 'Rien n'est sans raison' et 'Ce qui est sans raison', c'est penser une même chose, c'est-à-dire le rapport de mêmeté de l'être et de la raison, de l'être et du penser. Dans les deux cas, Heidegger fait voir et entendre que la raison n'est jamais seulement un rendre raison et que ce dernier opère comme voilement de la co-originarité de l'être et du penser. Tout comme pour le principe de raison, l'intonation de la définition de la gratuité se déplacerait : non plus 'Ce qui est sans raison', mais 'Ce qui est sans raison', dans la direction d'une correspondance entre être et raison, à méditer. Ainsi entendu, 'Ce qui est sans raison', la gratuité, ne renvoie plus à une spontanéité hors du champ de la rationalité ou à des raisons mal ren-

"Ratio", "raison ", traduit en allemand par "Grund ". "Dans l'ensemble, Grund évoque l'idée d'un domaine situé plus bas et qui en même temps porte, soutient. (...) Grund désigne le fond vers lequel nous descendons et auquel nous revenons, pour autant que le fond est ce sur quoi une chose repose, à quoi elle tient, d'où elle s'ensuit " (p. 211, souligné dans le texte).

25 P. 132. Cette dernière formulation pose problème. Comme le souligne Heidegger, on ne peut pas dire, à proprement parler, 'l'être est la raison'. Car l'être luimême n'est pas, dit Heidegger. "Ce mur qui se trouve devant vous et derrière moi 'est'. Il se montre à nous sans intermédiaire, comme chose présente » (p. 132). 
dues ${ }^{26}$. Elle renvoie comme son énoncé apparemment opposé, le principe de raison, à une méditation du rapport de résonance entre être et raison.

Sur le chemin de pensée du cours intitulé Le principe de raison, Heidegger donne à la sentence d'Angelus Silesius une fonction transitive vers le saut dans l'être en tant qu'être 27 . La rose sans pourquoi conduit à l'être, au sans-fond. Elle répond à la résonance de l'être. Elle est.

La référence à Jacques Derrida l'a évoqué, ce parcours interdit toute anthropologisation de la rose; il introduit plutôt à la structure ontologique du Dasein, là de l'être. Qui est la rose, dans la perspective de Heidegger? "Tout l'essentiel" de la sentence repose en ceci, écrit-il, qu'au "fond le plus secret de son. être l'homme ${ }^{28}$ n'est véritablement que s'il est à sa manière comme la rose - sans pourquoi » (p. 108). Selon Heidegger, le Dasein est, aussi, à sa manière, comme la rose, résonance à l'être. À sa manière, précise le penseur, car le Dasein est différent de la rose en ce qu'il a le pouvoir de se représenter la raison en tant que raison. Comment est-il quand il est comme la rose?

Dans un texte écrit peu après Sein und Zeit et qui porte aussi sur le fondement, Heidegger explicite la guise du Dasein en son acte de fonder en raison ${ }^{29}$. Il se ramifie en formes multiples. Trois, selon le penseur. Stiften, ériger, instituer, ébaucher un dessein; Boden-nehmen, prendre base, investi par l'existant; et begrunden, motiver, donner un fondement, fournir des raisons. Les deux premières ramifications énoncent la double forme de l'être-au-monde du Dasein : projetant-jeté. Dans l'ébauche d'un dessein, ce-qui-projette est commandé par le règne de l'être de-ce-qui-appelle et " est d'ores et déjà accordé à son ton" (p. 145). Dasein et monde adviennent co-originairement ${ }^{30}$. Le fonder comme projetant-jeté précède une

26 On peut ici penser à l'idée d'acte gratuit (ex. : un meurtre gratuit comme action arbitraire).

27 P. 143. "Le saut conduit la pensée, du domaine du principe de raison en tant que principe suprême concernant l'étant, à un dire qui parle de l'être en tant que tel ", p. $147-148$.

28 Je conserve ici la traduction de l'édition française.

29 «Le rapport qui, dès l'origine, unit liberté et fondement, liberté et 'raison', nous le désignons comme l'acte de 'fonder' (gründen) ", dans : "L'être-essentiel d'un fondement ou 'raison' " ( Vom Wesen des Grundes ", 1928), dans: Martin HEIDEGGER, Questions I. Paris, Gallimard, 1968, p. 144, souligné dans le texte. Je renvoie à cet article dans les paragraphes qui suivent.

30 Une autre image de cet acte de fondation serait celle de la Volonté de puissance de Nietzsche, telle que dite par Heidegger. La volonté advient en une émergence co- 
relation d'extériorité entre le Dasein et le monde, mais il permet un tel rapport. L'étant peut-il alors s'annoncer comme cause ou comme motif. D'où la possibilité du " pourquoi ", de l'intentionnalité, la troisième forme de l'acte de fonder, co-originaire aux deux autres. Elle surgit comme voilement de la notion de l'être, la "toute préliminaire réponse", "motivation première et dernière " (p. 150, souligné dans le texte). En sa forme authentique, la troisième ramification de l'acte de fonder n'appelle pas à fournir partout des raisons, mais à penser l'être, à méditer la mêmeté de l'être et de la pensée.

\section{Le Dasein}

peut, dans ses légitimations et justifications effectives, se débarrasser des fondements et 'raisons'; [il] peut réprimer la prétention de leur faire appel; [il] peut les renverser, les dissimuler (p. 151).

Les raisons, que le Dasein cherche, partout, à rendre, à fournir, à se donner, adviennent en tant qu'un dire, après coup, d'une réponse déjà donnée. Elles n'ont jamais rien fondé qui ne le soit déjà dans une résonance à l'être. Celui-ci "renferme déjà la réponse originelle, la réponse première et dernière, à toute question " (p. 150). Au fond le plus secret de lui-même, le Dasein n'est lui-même que s'il n'a pas échoué sur les raisons à fournir, à lui et par lui, déjà données; que s'il se laisse exister en résonance à ce don. Qu'il ait le pouvoir de se représenter la raison, cela l'appelle plutôt à penser l'être en tant qu'être.

Le chemin vers la gratuité signifie, dans ce projer, dans le projet de l'homme, l'épreuve d'un autre rapport à la raison qui n'échoue plus, toujours et partout, sur un rendre raison à la manière de la raison instrumentale. Elle signifie un laisser-être. Elle est écoute et réponse, les deux en même temps, à ce qui se donne déjà. La gratuité, cela arrive déjà toujours, mais l'homme la voile par la domination du penser sur l'être. Mais cela veut dire aussi que le Dasein existe déjà toujours comme la rose : comme résonance à ce qui se donne.

\section{Repenser gratuité(s)}

Je suis partie de l'hypothèse que femmes et hommes n'éprouvaient pas la gratuité de la même manière et avaient des défis différents à relever en regard de ses constructions culturelles. Guidée par Luce Irigaray, je me suis retrouvée en un air non familier - le plus familier? - où 'elle' n'est pas 
encore, mais joue de sa propre émergence à partir de la position, à elle, assignée par le texte. Avant de lire la méditation, par Heidegger, de la sentence d'Angelus Silesius, je voulais préparer une question qui ne nivellerait pas deux gratuités : celle d'elle et de lui. Qu'ai-je appris? Qu"elle' n'a pas de position énonciative dans le discours sinon l'archi-langage fondé sur la néantisation d"elle'. Qu'elle ne peut dire les gratuités, pas encore, sinon au mode conditionnel, dans le souffle de la préparation d'une respiration d'air. La tactique mise en jeu a fait apparaître que la perspective importe. Elle a soulevé nombre de questions. Est-ce que l'être, chez Heidegger, opère comme voilement d'un deuil de séparation d'avec 'elle'? Sédimente-t-il son oubli par une appropriation de l'autre qui enclôt l'homme dans son même, chaque fois répété?

Il construirait 'elle' comme gratuité même, mais 'elle' est aussi ailleurs, appel à un échange où elle pourrait être-avec l'autre ${ }^{31}$. Où elle pourrait, avec elles, avec eux, se tenir dans l'air libre?

'Lui' n'est pas gratuité. Il chemine vers elle. Il construirait ce chemin comme éveil à la correspondance entre être et penser.

L'homme cheminant vers le fond de la matière enveloppante de son être. Lui répondant, lui correspondant, en un jeu de résonances. Toujours déjà harmonisées? (p. 125)

Mais l'harmonie de l'être et du penser permet l'esquive de la différence sexuelle dans cette construction. L'attente, comme mode d'être du penseur, attend en direction du commencement des commencements, de la parole de Parménide : être et penser, le même. Pas le même?, demande Luce Irigaray 32 (p. 115). Être et penser, pas de même provenance? $\mathrm{Ne}$ puiseraient pas à une même ressource?

La pensée de l'oubli de l'être appelle au laisser-être; elle dévoile l'arraisonnement de la 'nature' par la pensée. Mais cela ne cache-t-il pas l'oubli d'un deuxième arraisonnement? D'elle'? De sa perspective, penser serait la construction par l'homme d'un monde pour lui,

un chemin, un projet, une traduction, qui réunit lui-même à lui-même, en son monde, sans alliance ni échange entre deux différents (p. 114)?

31. Comment le dire? Ce langage, toujours le sien? « Une langue qui ne serait pas la sienne »? (p. 99).

32 P. 115. "Laisser être doit-il s'entendre comme laisser se déployer la pensée de l'homme, ou laisser s'épanouir la nature? Ces deux avènements peuvent-ils arriver dans le même temps? " (p. 23) 
Était-il déjà trop tard au commencement du destin de l'être?

'A droite les garçons, à gauche les filles' (Parménide). Entre eux, la fracture de deux univers qui ne se parlent plus l'un à l'autre (p. 120).

Il y aurait deux mouvements, presque contraires l'un à l'autre. 'Elle' demeure disponible, toujours, pour la venue de l'être et du rien. "Ne prenant rien, gardant le tout déposé-disposé dans ou sur elle, prêtant son corps vivant comme lieu de rassemblement du tout " (p. 63). 'Lui' attend, disponible, lui aussi dans "la 'maison de l'être "; il correspond à l'appel premier lancé vers lui, comme " jetant-projetant".

Le Da-sein possède la structure ontologique de la projection. Il devance. $\mathrm{Si}$ la phusis, quasi maternellement et avant toute dénomination, est tournée vers le futur en tant qu'elle donne lieu à la croissance, l'homme doit se projeter vers le futur pour ne pas régresser vers ce qui lui a donné à vivre. La femme génitrice, elle, ne découpe pas un horizon en se tournant vers l'avant, elle se donne en avant, et se confond à ce qui se donne à partir d'elle. Dans cette indifférenciation temporelle d'elle et de l'autre, qu'elle sous-tend et accompagne, elle offre matière à l'ek-stase du temps. N'y existant pas, elle-même comme sujet (p. 95).

Il y aurait deux chemins à parcourir pour pouvoir vivre, ensemble, dans l'air, hors du réceptacle. 'Elle' peut tactiquement détourner la position, à elle assignée, de gratuité. Elle peut éprouver le projet de penser la différence sexuelle et appeler à un échange dans un changement d'air. Ce ne serait plus gratuité? Ou le "sans prix " incommensurable, pour les unes et pour les unes? 'Lui' peut également détourner tactiquement sa position en la maison du langage. Martin Heidegger n'a jamais prétendu autre chose que de préparer une autre pensée. 